\title{
Multi wavelength investigation of the eruption of a sigmoidal quiescent filament ${ }^{\star}$
}

\author{
Ch. Marqué ${ }^{1}$, P. Lantos ${ }^{1}$, and J. P. Delaboudinière ${ }^{2}$ \\ 1 DASOP-LPSH, Observatoire de Meudon, 92195 Meudon Cedex, France \\ 2 Institut d'Astrophysique Spatiale, Université Paris Sud, 91405 Orsay, France
}

Received 19 November 2001 / Accepted 18 February 2002

\begin{abstract}
We report the first observation of a filament eruption in the metric and decimetric range, where the behavior of the filament can be followed during the event via thermal radio emission diagnostics. The event, occurring on February 28th 2001, involved a quiescent filament in a sigmoid magnetic configuration, whose eruption is triggered by the birth of a small parasitic polarity. Faint radio bursts mark the beginning of the event, which shows the appearance of a brightness temperature depression associated with the filament seen in EIT, and its propagation on the disk up to the limb. The event is associated with a halo CME observed with the coronagraphs LASCO C2 and C3, which shows a significant spatial and temporal continuity with the radio observations. Finally, static dimmings, similar to what is currently observed in EUV or SXR domains, are also detected in the radio band, around the site of the eruption. Movies of the event are attached to this article.
\end{abstract}

Key words. Sun: corona - Sun: filaments - Sun: Coronal Mass Ejections (CMEs) - Sun: radio radiation

\section{Introduction}

The relationship between filament eruptions and CMEs has been considered since the early studies of the large coronal transients and confirmed by long-term observations of the solar activity with solar missions such as SMM (Webb \& Hundhausen 1987) or sOHO. Nevertheless, coronagraphs, with their large occulting disk, cannot observe the CME onsets, which are likely to occur in the lower corona.

With the launch of coronal imagers in the EUV or SXR domains, for example SXT on board YOHKOH, it has been possible to detect on the disk, first the eruptive processes related to the ejection, involving both active regions or quiescent filaments, as well as the coronal restructuring during and after the event. Some magnetic configurations such as sigmoids (Rust \& Kumar 1996) are now considered as possible predictors of solar eruptions (Canfield et al. $2000)$, provided that the notion of sigmoids is clearly defined (Glover et al. 2000). The restructuring of the corona is also indicated by the occurrence of dimmings around the site of the eruption. After their discovery during the SKYLAB era (Rust \& Hildner 1976), dimmings associated with

\footnotetext{
Send offprint requests to: Ch. Marqué, e-mail: christophe.marque@obspm.fr

* The movies are available in electronic form at http://www.edpsciences.org
}

coronal mass ejections are now currently observed both with the YOHKOH/SXT and SOHO/EIT imagers (Sterling et al. 2000).

Radio observations of filament eruptions and CMEs at long decimetric and metric wavelengths generally show non-thermal radio emissions indicating the site of particle accelerations, or the propagation of coronal disturbances (see Pick Pick 1999 for a review). In contrast, low energetic events, without strong radio bursts or EUV emissions, allow us to see the thermal radio counterpart of the coronal restructuring, as shown in a previous study (Marqué et al. 2001).

In this article, we present a multi-wavelength analysis of an event which occurred on February 28th 2001, involving a quiescent filament embedded in a sigmoidal magnetic configuration. Faint radio bursts mark the beginning of the event, which is followed from the erupting site up to 25 solar radii in the LASCO-C3 coronagraph. Using the Nançay Radioheliograph (Kerdraon \& Delouis 1997), we can study the onset of the eruption as well as the motion of the filament, seen as a brightness temperature depression, compared to the thermal emission of the corona. EUV data were provided by the SOHO/EIT imager (Delaboudinière et al. 1995), and coronagraphic data by the soHo/LASCO instrument (Brueckner et al. 1995); Xray flux was given by GOES satellite via the SPIDR data center. We used an $\mathrm{H} \alpha$ observation from the Kanzelhöhe 


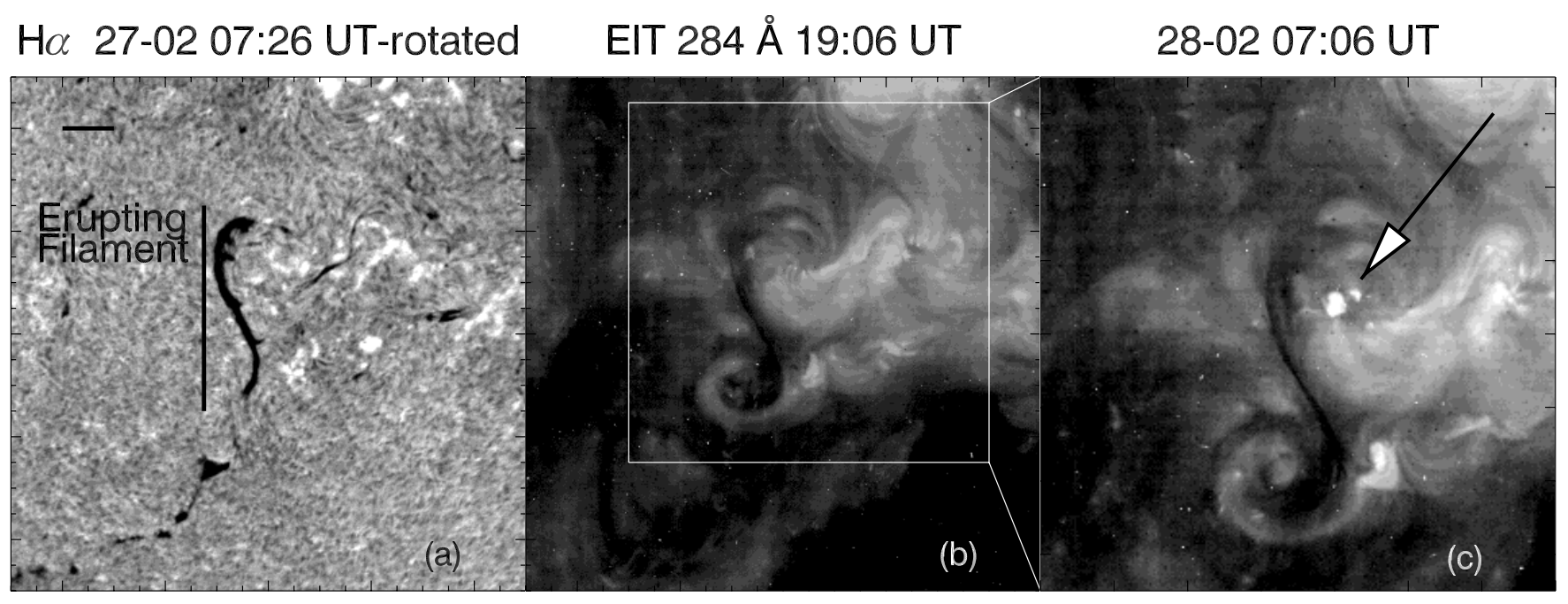

Fig. 1. a) $\mathrm{H} \alpha$ Kanzelhöhe observation of the erupting filament on February 27th. The part of the filament to disappear is indicated by the vertical thick line. Horizontal stroke is a scale length of $100^{\prime \prime}$ that applies also for the next panel. b) EIT map at $284 \AA$ (Fexv) showing the overlaying magnetic configuration. The filament is seen embedded, in absorption, within the magnetic arcades. c) Close up view of the area contained within the square drawn on b), on February 28th. The arrow points out the emergence of the small bright point, which lies above a parasitic polarity, compared to the surrounding mean magnetic field.

observatory, on February 27th, for the pre-eruptive study. Three movies of the event are available with the electronic version of this article: the first one from the EIT instrument, in the Fe XII band, the second one displays the radio observations at $410.5 \mathrm{MHz}$, and the last one is a composite movie of LASCO-C2 and Nançay data at $410.5 \mathrm{MHz}$, illustrating the continuity between radio and coronagraphic observations. Aliasing effects, due to the lack of short baselines, are visible in radio data and explain why multiple images of the Sun overlap.

\section{Data}

\subsection{Description of the event}

On February 28th 2001, after 13:00 UT, a filament located West $05^{\circ}$ South $20^{\circ}$ erupted with minor energy release signatures, revealed by faint and short duration non-thermal radio emissions and a weak Long Duration Event (hereafter LDE) reaching the B4 level in the 1-8 $\AA$ band of GOES. A faint halo CME was observed after 14:50 UT in LASCO coronagraphs $\mathrm{C} 2$ and $\mathrm{C} 3$ with a moderate velocity $\leq 300 \mathrm{~km} \mathrm{~s}^{-1}$.

\subsubsection{The pre-eruptive evolution}

The filament was observed in $\mathrm{H} \alpha$ before its disappearance at Kanzelhöhe observatory on February 27th. Figure 1a shows that it is the leading part of a long medium-latitude filament, located in the neighborhood of a small spotless active region, west of the filament. While the trailing part of the filament is a long string of small dark components, the erupting one is a stand-alone structure, which reveals feet on its sides. In the following, only the erupting part will be studied. One day before its eruption, the filament appears as a S-shaped structure, in a sigmoid configuration, both in $\mathrm{H} \alpha$ and in the FeXII band of the EIT instrument. Figure 1b shows, in the Fe Xv band, that the overall magnetic structure is also sigmoidal, and that the filament is surrounded by non-potential arcades. Radio observations prior to the eruption, not shown here, do not reveal any counterpart to the erupting filament itself, due to the neighborhood of unresolved loops associated with the small active region, and probably to its low altitude (the filament does not appear clearly on East limb in He II images between February 20th and 22nd).

The evolution of the filament, close to the eruption, seems to be related to the nearby small active region. The comparison of EIT images on February 27th and February 28th, a few hours before the event, reveals the appearance of a small bright point, pointed out by the arrow in Fig. 1c. SOHO/MDI magnetograms indicate it is a parasitic polarity compared to the main surrounding magnetic field.

\subsubsection{The eruption}

The main steps of the event are presented in the following section. Figure 2 provides an exhaustive display of the time sequence. As already mentioned, the energy release, both in radio and soft $\mathrm{X}$-ray band, is weak; the event is associated with short duration non-thermal radio emissions and with a $\mathrm{LDE}(\sim \mathrm{B} 4)$ beginning at 13:20 UT, and lasting around 5 hours.

The first manifestation of the event is a group of faint radio bursts, occurring at four frequencies, 164, 236.6, 327 , and $410.5 \mathrm{MHz}$ (hereafter burst A) between 11:18 and 11:23 UT. The temporal evolution of the burst is revealed by high time resolution images, and is plotted on upper left panel of Fig. 2. Two kinds of emissions 


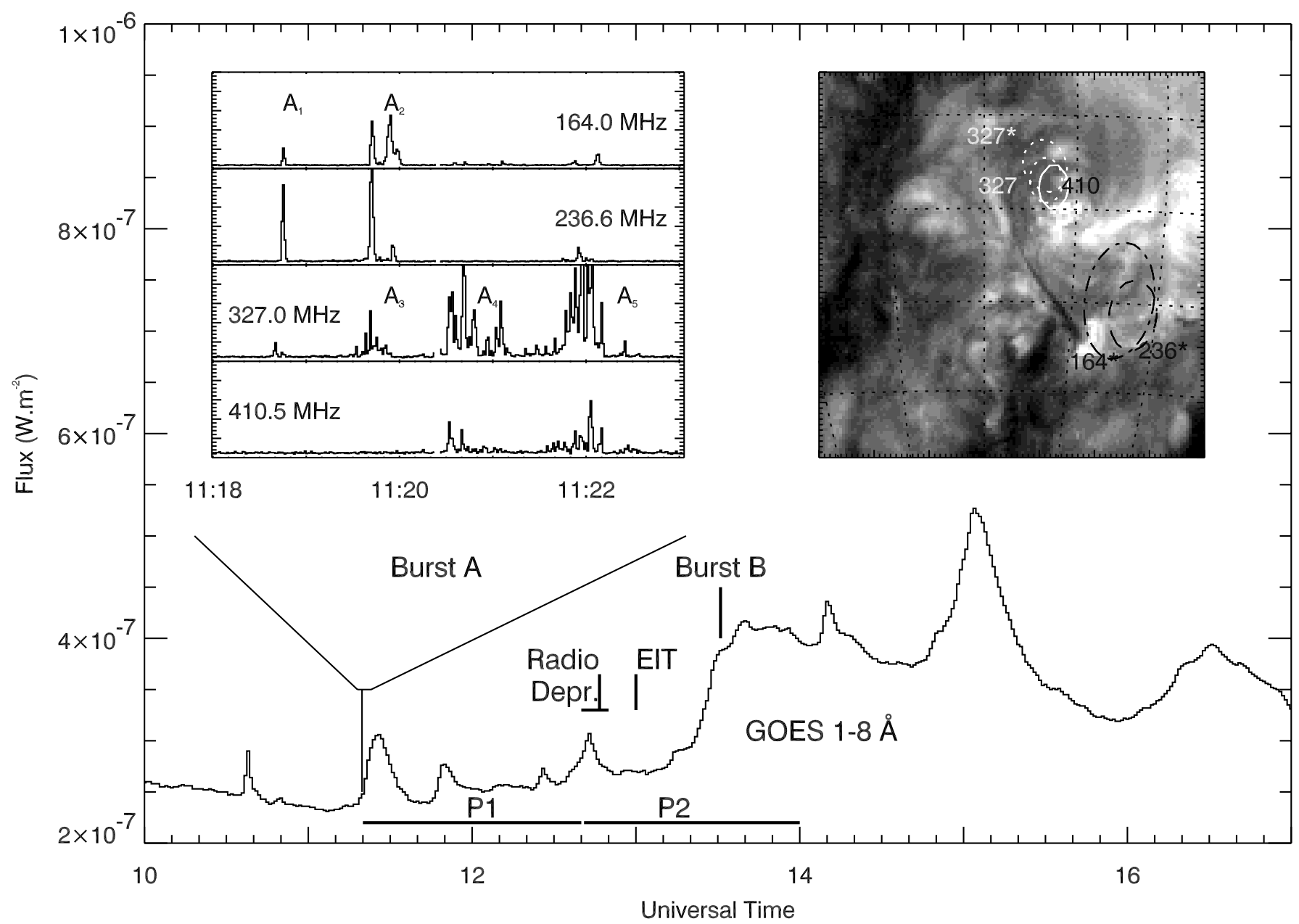

Fig. 2. Synoptic presentation of the event. The main curve is the GoEs flux in the range $1-8 \AA$. Two radio non thermal emissions are indicated as burst $\mathrm{A}$ and burst $\mathrm{B}$. The two steps of the eruption are indicated as P1: slow evolution phase, and P2: eruptive phase. The approximative time of the radio depression appearance is pointed out, as well as the first EIT image of the eruption. Upper left panel: time profile of the peak brightness temperature of burst $A$ at four frequencies. Different bursts are called $A_{1} \ldots A_{5}$. Right panel: locations of radio emission sites; contour plots are drawn for each frequency at $90 \%$ of the peak brightness, at two hours; 11:19:40 UT(star) and 11:20:30 UT

can be distinguished: a first group of low frequency and short duration bursts (called $A_{1}$ and $A_{2}$ ) occurred between 11:18-11:20 UT, at 164 and $236.6 \mathrm{MHz}$, probably faint type III bursts. At high frequency, faint continua, with narrow band short duration bursts are detected between 11:19-11:23 UT $\left(A_{3}, A_{4}\right.$ and $\left.A_{5}\right)$. The weakness of these emissions does not allow a precise spectral type determination, but the low and high frequency emissions have distinct time profiles. Locations are also different: while high frequency bursts occurred north to the filament, in the vicinity of the small parasitic polarity (bright point seen in EIT/Fe XV and pointed out by an arrow in Fig. 1c), the low frequency emissions are located both south west to the filament $\left(A_{1}\right.$, and first peak of $A_{2}$ at 164 and $236.6 \mathrm{MHz}$ and second peak at $236.6 \mathrm{MHz}$ ) and in the vicinity of the parasitic polarity (second peak of $\mathrm{A}_{2}$ at $164 \mathrm{MHz}$ ). The upper right panel of Fig. 2 displays the two sites of nonthermal emission at two different times.

The eruptive process can be divided into two steps: a slow evolution phase and the filament eruption itself (resp. phases $\mathrm{P} 1$ and P2 indicated in Fig. 2). The non-thermal radio emissions mark the beginning of the phase $\mathrm{P} 1$. As seen in the Fe XII band of the EIT instrument, the high frequency continua are associated with faint brightenings in the small parasitic polarity between 11:12 and 11:36 UT. The south part of the filament slowly begins to expand after the type III like burst occurrence (as observed at 11:36 UT in EIT).

Being observed in $\mathrm{H} \alpha$ as a stand-alone feature, the filament (see Fig. 1a) could erupt as a single structure. Nevertheless, the south and the north part of the filament evolved in different manners.

The event is illustrated in Fig. 3 which shows, in particular, the evolution of the south part. It can be divided into a long S-shaped structure (pointed out by a white thick head arrow in Fig. 3), ended by a thin curved filamentary feature. This system slowly expanded from the time of the radio burst (metric component) and interacted with overlying large scale loops whose feet were seen in EIT after 12:36 UT (pointed out by thin head arrows in Fig. 3 


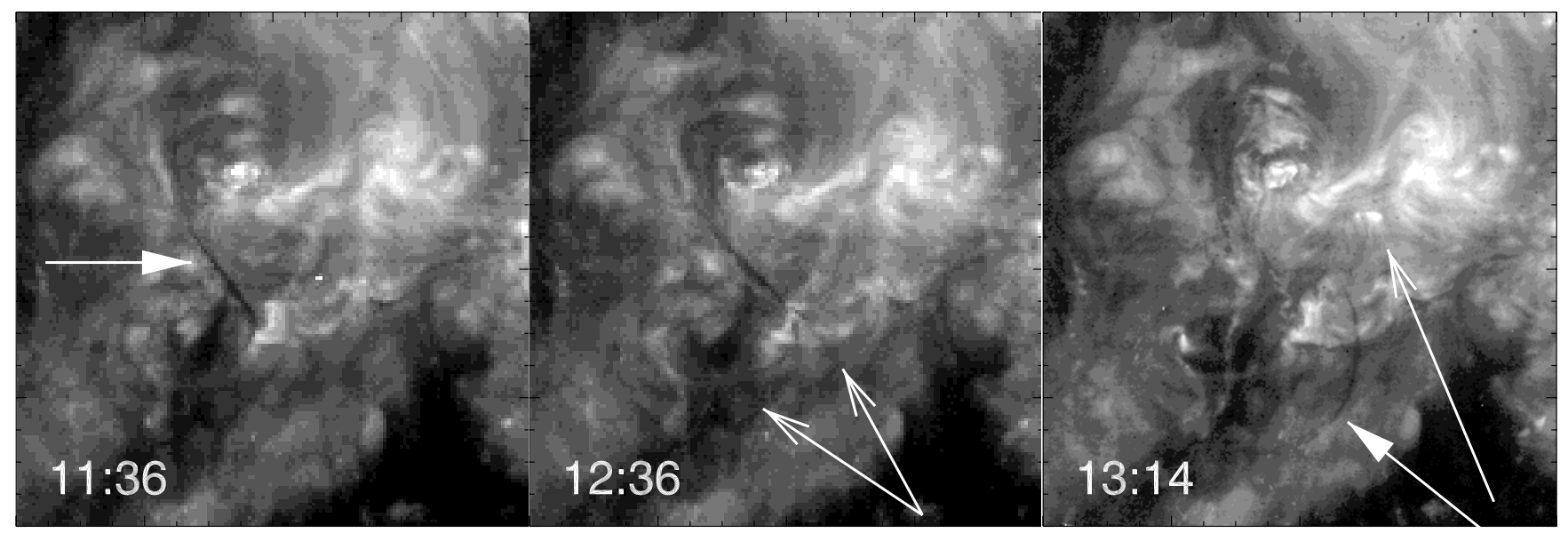

Fig. 3. Observations of the filament neighborhood at $195 \AA$ (Fe XII). Three different steps of the event are shown here. On left hand panel, the pre eruptive state, just after radio burst A occurred (see Fig. 2); this marks the beginning of the expanding motion of the filament; the main body of the erupting filament is indicated by the thick head arrow. The middle view illustrates the interaction, during the slow ascending phase, with the overlying loops in regions pointed out by white arrows. On right hand panel the eruption is underway; the filament is indicated by the thick head white arrow, and the foot of the overlying arcades by simple arrow.

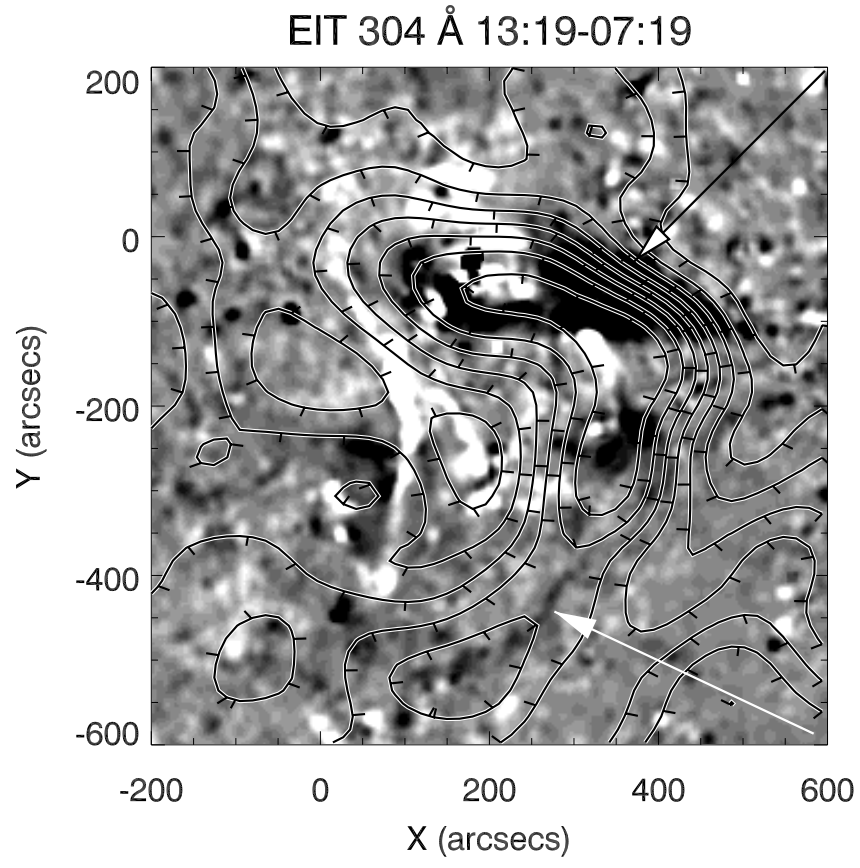

Fig. 4. He II band observations, using the image difference method, at the time of the eruption, 13:19 UT. The south part of the filament (white arrow), as well as the large structure in absorption in the north part (black arrow with white head) are indicated. Radio contours at $410.5 \mathrm{MHz}, 13: 19 \mathrm{UT}$, are overplotted with inward tick marks for brightness depressions. The field of view is identical to Fig. 3 .

at 13:14 UT). At this time, these loops expand with the extreme southern feature. The eruptive phase, indicated as P2 in Fig. 2, begins between two EIT/Fe XII images, 12:36 and 13:14 UT.

The northern part of the filament is embedded in overlying arcades that are seen to be shaken during the erup- tion. No structure in absorption erupts at $195 \AA$ (Fe XII) after 13:00 UT, at this location. In the He II band, an area appears in absorption west of the initial location of the filament at 13:19 UT (Fig. 4). This structure can easily be observed by subtracting the previous image at 07:19 UT. One image only is available at this wavelength at the time of the eruption; this does not allow us to determine the evolution of this dark pattern in the EUV band; nevertheless, it is certainly the north part of the filament, as indicated by quick-look $\mathrm{H} \alpha$ data (movie file) from HASTA observatory. Being observed only in the He II band of the EIT instrument, this suggests that its altitude is, at this time, lower than the southern part described earlier.

Radio observations precise the eruptive behavior of the entire system. Figure 5 shows different steps of the radio event. After 12:40 UT, a depression is seen to appear and expand along the main body of the EUV filament. It is observed in the four radio bands of the NRH, suggesting a large altitude range. At this stage, the depression is subdivided into three main dips that evolved in different manners.

The southern one (F1) is spatially associated with the southern part of the eruptive filament presented in the last panel of Fig. 3 (thick head arrow). Short baselines are missing at the time of the observation, and thus an absolute flux determination is impossible. Nevertheless, brightness temperature depressions, relative to the quiet sun on the disk, are reliably measured; they are about $-(5.5-3.5) \times 10^{5} \mathrm{~K}$ at $410.5 \mathrm{MHz}$ and $-(3-1.5) \times 10^{5} \mathrm{~K}$ at $236.6 \mathrm{MHz}$. The motion of this structure can be followed on the disk and beyond the optical limb (see image at 14:11 UT in Fig. 5). This depression vanishes after $\sim 14: 30 \mathrm{UT}$, a long time after the filament disappeared in the FeXII band of EIT (13:30 UT). During its eruption, both on the disk and on the limb, the radio counterpart 


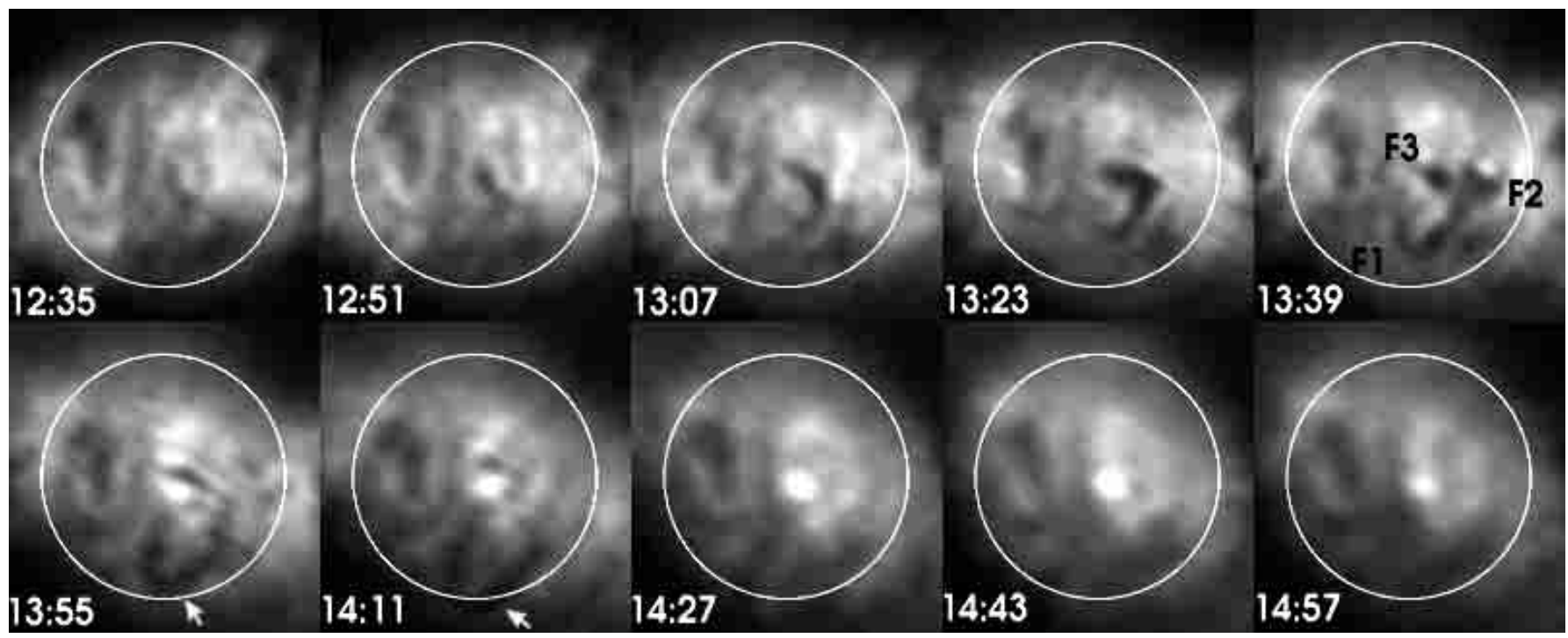

Fig. 5. Radio observations of the eruption in the decimetric range at $410.5 \mathrm{MHz}(73 \mathrm{~cm}-\lambda)$; the white circle indicates the optical limb. The expansion of the radio depression associated with the filament is shown here, up to the limb (pointed out by white arrows on lower images, at 13:55 and 14:11 UT). The area covered by dips F2 and F3 reaches a maximum extension between 13:23 and 13:39 UT.

of the filament is seen as a depression. The mean projected velocity is about $100 \mathrm{~km} \mathrm{~s}^{-1}$.

Two other depressions are observed. A large dip (called Dip F2) west of the erupting site is observed on radio images, at all frequencies, and starting from the initial position of the filament. Brightness temperatures are extremely low within this depression, compared to the mean brightness temperature of the quiet sun; the depth of the dip is about $-(7-5) \times 10^{5} \mathrm{~K}$ at $410.5 \mathrm{MHz}(13: 22 \mathrm{UT})$ and $-(5-4) \times 10^{5} \mathrm{~K}$ at $236.6 \mathrm{MHz}$. This depression has a westward motion observed up to the limb, and has no obvious EIT counterpart in the Fe XII band. Nevertheless, it is partially cospatial with the structure observed in the He II band and pointed out by the white head black arrow in Fig. 4; the orientation is roughly similar, but the shape is different. The last depression (Dip F3) is located north to the filament site. It appears in the early stages of the radio eruption, as an appended part of dip F2, but remains static up to $13: 31$, when a faint burst occurs at $410.5 \mathrm{MHz}$ (burst B in Fig. 2). Then, it disappears northward in about $50 \mathrm{~min}$. The area covered by the dips $(\mathrm{F} 3+\mathrm{F} 2)$ reaches a maximum extension around 13:30 UT; at $410.5 \mathrm{MHz}$, it extends from $0^{\circ}$ to $45^{\circ}$ in longitude, and from $-10^{\circ}$ to $+10^{\circ}$ in latitude.

Other coronal manifestations are triggered by this filament eruption. Near the location of the initial filament, transient coronal holes are forming after 12:55 UT in YOHKOH/sXT images, enclosed by a bright sigmoid structure. In EIT, one of the holes south of the filament is also visible, in the early steps of the eruption, at 13:13 UT (the northern one is not detected due to expanding flare loops appearing during the event). This south dimming slowly expands eastward and southward until $\sim 20: 00$. Similar dimmings are also observed with difference image techniques applied to radio data; they are clearly different from the moving dips already discussed (Fig. 5). Figure 6 shows the locations of the dimmings in radio and in the Fe XII band. Pre and post eruptive images have been subtracted in order to enhance the brightness differences. Dimming positions are roughly similar in both domains, but radio brightness depressions extend over a larger area (for example D1 or D3), and seem to connect different EIT dimmings (D2 and D4).

Finally, the present event is associated with a CME observed with the SOHO/LASCO coronagraphs. This is a faint halo CME, appearing first at 15:50 UT in the LASCOC2 field of view, with an angular size of $215^{\circ}$ and mostly visible on the west side of the field of view (see left panels of Fig. 7). The mean projected velocity of the leading edge is lower than $300 \mathrm{~km} \mathrm{~s}^{-1}\left(278 \pm 4 \mathrm{~km} \mathrm{~s}^{-1}\right.$ at a position angle of $\left.240^{\circ}\right)$.

Structures are observed within the CME pattern. Due to the halo geometry that induces projection effects, it is nevertheless rather difficult to make a correspondence with the radio depressions. In such an attempt, the location of the southern radio depression was compared to those of the inner Leading Edge (LE) and Trailing Edge (TE) of the CME. Results presented on right panel of Fig. 7 show the good correlation between the radio and the coronagraphic structures, but the uncertainties do not allow a one to one association. However, the leading and the trailing edge of the radio depression and of the CME can be fitted to common trajectories. This suggests that both signatures belong to the same evolving structures.

\section{Interpretation and discussion}

\subsection{The radio depressions}

Radio depressions are totally new features observed during CMEs. Studies such as the present one should help us to 
EIT $195 \AA \AA 15: 24-12: 00$ UT
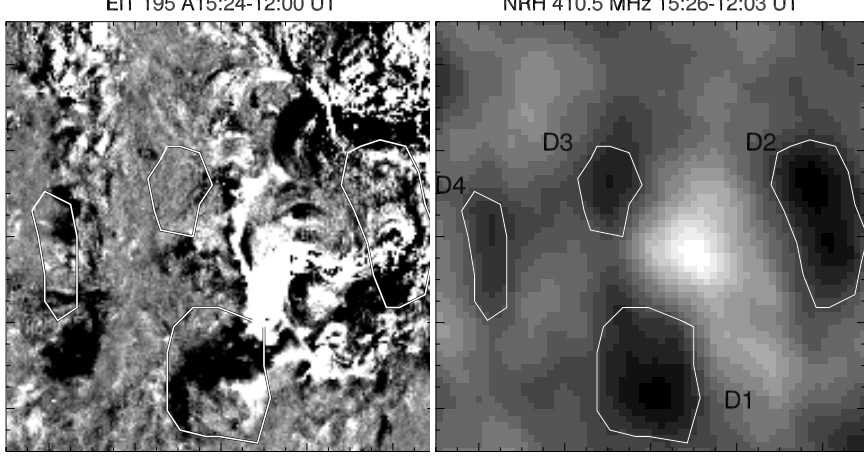

Fig. 6. Comparisons of dimming locations in radio $(410.5 \mathrm{MHz}$, right image), denoted D1,D2,D3 and D4, and in the Fe XII band $\left(1.5 \times 10^{6} \mathrm{~K}\right)$. For the EUV images the solar rotation was taken into account. The borders of the radio dimming area are plotted on each image.

understand the nature of the radio depression expanding from the site of the eruption.

The south depression, as illustrated in Fig. 4, is obviously related to the EUV filament. Contrary to millimetric and centimetric radio ranges, where filaments have been extensively studied for years, brightness temperature depressions in the long decimetric range were observed recently for the first time, with the Nançay Radioheliograph (Delouis 1999; Marqué et al. 1999). The nature of the detected depression is an important question regarding the analysis of the filament coronal environment.

In the following, we first try to estimate the effect of the beam convolution upon the observed brightness temperature. As the size and the shape of the filament was the same in the four EIT bands, we took as a model the filament observed by EIT in the HeII band, embedded into a synthetic corona whose brightness temperature is $9 \times 10^{5} \mathrm{~K}$. We also included a gaussian distribution of temperature fluctuations whose amplitude is about $1 \times 10^{5} \mathrm{~K}$. We arbitrarily give a brightness temperature to the filament of $7 \times 10^{5} \mathrm{~K}$ in a first time, and then decrease it to $10^{5} \mathrm{~K}$ and $0 \mathrm{~K}$. We made calculations including different sizes of the filament, in order to estimate the effect of a possible hot layer surrounding the filament. We consider 4 different widths in terms of EIT pixels $\left(1\right.$ pixel $\left.=2.6^{\prime \prime}\right)$, $2,5,10$ and 20 (the width of the filament in EIT images is smaller or equal than $10 \operatorname{arcsec} \sim 4$ pixels). Resulting maps were then convolved by the beam of the NRH, whose FWHM size is about $35 \times 55$ arcsec at $410.5 \mathrm{MHz}$, producing images shown in Fig. 8. The different results are presented in Table 1.

For the south part of the filament, dips are too faint, in the first two cases, compared to the measurements $\left(\Delta T_{\mathrm{F}-\mathrm{QS}} \sim-(3-5) \times 10^{5} \mathrm{~K}\right)$. Extreme parameters (bold type values in Table 1) are required to explain the observed brightness depression: a $0 \mathrm{~K}$ brightness temperature and/or a fourfold size structure compared to the observed filament. This suggests, in this case, that the radio emission does not come from a cold medium of the size of the filament.
The same convolution was performed for the north part of the depression, assuming a constant size. Depressions obtained are about $-2 \times 10^{5} \mathrm{~K}$ for an initial brightness temperature of $7 \times 10^{5} \mathrm{~K}$, and $-7.3 \times 10^{5} \mathrm{~K}$ for a brightness temperature of $10^{5} \mathrm{~K}$. The north depression, observed in the He II band, probably associated to filament material, is a large structure, compared to the beam of the telescope. In this case, a cold sheet of material with a brightness temperature of $10^{5} \mathrm{~K}$, could be compatible with our measurements $\left(-(7-5) \times 10^{5} \mathrm{~K}\right)$. We need now to check the physical relevance of such a cold structure.

Filaments are known to be surrounded by a layer called the Prominence Corona Transition Region (PCTR). Chiuderi Drago (1990) made a comparison between the Differential Emission Measure (DEM) of filaments in EUV and the radio spectrum up to $20 \mathrm{~cm}-\lambda$. Taking into account the orientation of the magnetic field with respect to the temperature gradient, she established a model of the transition region emission in radio band from the millimetric to the long centimetric domain. It is possible to extend her model to longer wavelengths and to estimate the brightness temperature we would expect. With a pressure of $p=0.2 \mathrm{dyn} \mathrm{cm}^{-2}$ and $\theta=80^{\circ}$ (angle between the temperature gradient and the direction of the magnetic field), and a coronal electronic temperature of $1.5 \times 10^{6} \mathrm{~K}$ we get a brightness temperature of $\sim 7.7 \times 10^{5} \mathrm{~K}$ at $410 \mathrm{MHz}$ $(\sim 70 \mathrm{~cm}-\lambda)$. The intrinsic brightness temperature of the PCTR is about $3.7 \times 10^{4} \mathrm{~K}$. This means that the total brightness temperature is mostly dominated by the emission of the overlaying corona, and that the PCTR cannot be easily probed. Assuming a hydrostatic corona with an initial density of $n_{0} \sim 4.54 \times 10^{8} \mathrm{~cm}^{-3}$ (Allen 1947), the resulting brightness temperature, outside of the filament area is about $6.7 \times 10^{5} \mathrm{~K}$; in this case, the radio filament should be, by contrast, observed in emission. With a twofold higher density at the base of the corona, a depression of about $-6 \times 10^{5} \mathrm{~K}$, compared to the quiet sun level, appears by contrast. Thus, a depression cannot be distinguished without a drop of the coronal density above the filament. The pressure equilibrium at the PCTR/corona border settles the electronic coronal density above the location of the filament, and may lead to depressions if the surrounding coronal density is higher.

A drop in the electronic density must be achieved in order to probe the filament environment. A cavity is a natural way to do this. Cavities are known to exist in the vicinity of filaments in white light during eclipses or in Xray observations (Saito \& Tandberg-Hanssen 1973; Vaiana et al. 1973). The density in such structures is thought to be 2 to 4 times lower than in the surrounding corona, as indicated by Engvold (1988). We now estimate the effect of such drops in the coronal density for the resulting brightness temperature as follows: we assume a spherically symmetric corona, with simple electronic density profiles derived from Allen (1947) and Saito et al. (1977) and a gaussian depression. Parameters were the electronic temperature $T_{\mathrm{e}}$ of the corona, taken as $10^{6}, 1.5 \times 10^{6}$ and $2 \times 10^{6} \mathrm{~K}$, the depth of the density hole 0.5 , or 0.75 , and 

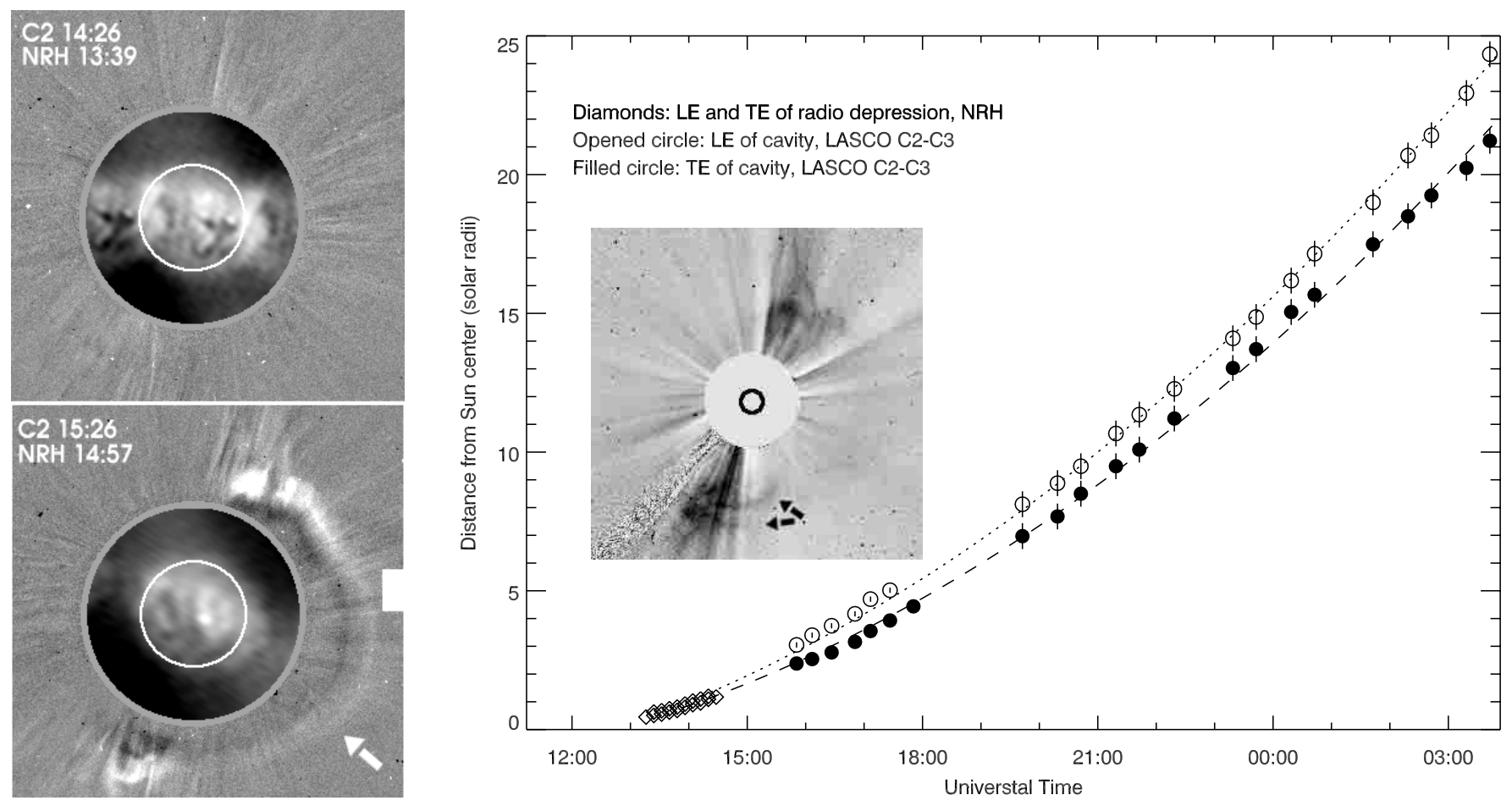

Fig. 7. Left panel: upper image presents a pre CME high solar corona. The lower image shows the expansion of the halo CME seen in LASCO-C2 coronagraph, and pointed out by the white arrow. The CME is mainly expanding westward as expected by the orientation of the filament. Right panel: height/time plot of the structure pointed out in the inner C3 image with black arrows, compared to the evolution of the radio depression associated with the filament.

the frequency 236.6 and $410.5 \mathrm{MHz}$. Results are displayed in Fig. 9. They are in good agreement with measurements. A twofold drop in density, with a moderate electronic temperature, can explain the depression associated with the south part (SF) of the filament (F1 in Fig. 5), at both frequencies. The large dip associated with the north part of the filament can also be explained with a twofold drop, assuming a higher temperature.

In summary, a cavity seems to be the easiest way to explain the radio depression (F1 in Fig. 5) associated with the south part of the filament. As the radio depression is seen when the filament propagates through the corona, we infer that this cavity is linked to the filament, and related to the magnetic structure that supports the filament against gravity, for example a flux tube (see for example Aulanier \& Démoulin 1998).

For the northern part, a drop in density is also necessary; it can be achieved via a cavity or a local perturbation of the coronal density compared to the surroundings. Radio depression may finally become more pronounced due to the eruptive behavior of the system. First, if we assume that the cavity exists before the eruption, it should inflate as the filament comes through the upper corona; the density inside the cavity should then decrease, allowing the depression to be detectable in the radio range (for example dip F1). On the other hand, the overlaying corona can be evacuated above the site of the eruption, allowing radio waves to reach layers near the PCTR and thus explaining the very low brightness temperatures within dips F2 and F3 at $410.5 \mathrm{MHz}\left(-(7-5) \times 10^{5} \mathrm{~K}\right.$ compared to the quiet sun level), and associated with the large feature observed in the He II band.

\subsection{Coronal manifestations of CME onsets}

In this section we will discuss both the possible triggering and the coronal manifestations of the eruption.

From an observational point of view, the relationship between magnetic flux emergence and filament eruptions is well known (see for example Feynman \& Martin 1995). Theoretical work has been done to explain such correlations (Chen \& Shibata 2000) using 2D magnetic structures. Although it is difficult to put a peculiar event in a class of theoretical models, many signatures are observed. Contrary to a previous observation, where non-thermal emissions marked the onset of the eruptive phase (Marqué et al. 2001), burst A points to the onset of the slow outgoing motion (phase P1 in Fig. 2). The locations of the bursts, on one side of the filament, and near the parasitic polarity, suggest a reconnective process occurring between this polarity and the overlaying arcades. The energy release evolution is rather classical but at a low level, as seen with GOES. Flare loops observed with EIT link the vicinity of the parasitic polarity and the area across the inversion line as expected. A faint non-thermal polarized radio source appears about one hour after the beginning of the 
Table 1. Brightness temperature depressions $\left(\times 10^{4} \mathrm{~K}\right)$ resulting from the convolution of different size filaments by the beam of the telescope at $410.5 \mathrm{MHz}$. Three different brightness temperatures are assumed for the filament (left column).

\begin{tabular}{lcccc}
\hline \hline Fil. Width (pixels) & 2 & 5 & 10 & 20 \\
\hline$T_{\text {Bfil }}=7 \times 10^{5} \mathrm{~K}$ & -1 & -3 & -5 & -9 \\
$T_{\text {Bfil }}=1 \times 10^{5} \mathrm{~K}$ & -4 & -10 & -16 & $-\mathbf{3 0}$ \\
$T_{\text {Bfil }}=0 \mathrm{~K}$ & & & & \\
\hline
\end{tabular}

eruption (see lower left image of Fig. 5) at $410.5 \mathrm{MHz}$, above the site of the flare loops. This non-thermal emission appears roughly $15 \mathrm{mn}$ later at $164 \mathrm{MHz}$. Considering a hydrostatic corona with a scale height $z_{h} \sim$ $0.07 R_{\odot}$ and the critical densities $\left(n_{164} \sim 3.3 \times 10^{8} \mathrm{~cm}^{-3}\right.$ and $n_{410.5} \sim 2.08 \times 10^{9} \mathrm{~cm}^{-3}$ ), the ascending velocity of loops supporting the radio emission should be about $100 \mathrm{~km} \mathrm{~s}^{-1}$.

Since the launch of coronal imagers in soft X-rays and EUV domains, it has been possible to follow the onset of Coronal Mass Ejections on the disk, associated or not with active regions. Hudson (1999) summarizes the different manifestations of CME onsets, essentially in the frame of $\mathrm{YOHKOH} / \mathrm{SXT}$, but these phenomena are also observed in the EUV domain. Taking into account the characteristic cooling time, dimmings are certainly the signatures of mass outflows, expelled during the eruption (Hudson et al. 1996); a recent spectroscopic observation of a dimming related to a CME was reported by Harrison \& Lyons (2000), who concluded that the dimming was due to a density decrease in the corona. Some of these dimming events are related to pre-flare sigmoid structures, which are supposed to be progenitors of eruptions in the frame of space weather researches (Canfield et al. 2000). The event under study can be placed in this framework, as the pre-event state is a "dark" sigmoid structure; there are dimming signatures in EUV and radio, and finally a halo CME is visible in the LASCO-C2 coronagraph. Although the energy release signatures are small here, the coronal disturbances have very broad sizes. In a synoptic study of 11 halo CMEs, Hudson et al. (1998) had already found that 7 were related to sigmoid structures, and were associated with flares ranging from A1 to $\sim \mathrm{M} 1$ in the GOES classification. Such low energy release events are interesting especially in the radio range, as the thermal counterpart of the corona can be probed during the eruption in the absence of strong radio bursts. The dimming areas observed in this event are compatible with mass loss $\Delta m \geq 1.2 \times 10^{13} \mathrm{~g}$ (total of mass deduced from $\mathrm{D} 1+\mathrm{D} 2+\mathrm{D} 3+\mathrm{D} 4$ as indicated in Fig. 6), which is a very small fraction $(\sim 1 \%)$ of the typical CME mass (Howard et al. 1985; Vourlidas et al. 2000).

The combined observations of the EIT instrument together with radio data may help us to understand the eruptive motion of the dark sigmoid, in particular, the asymmetry between the north and the south part. It may

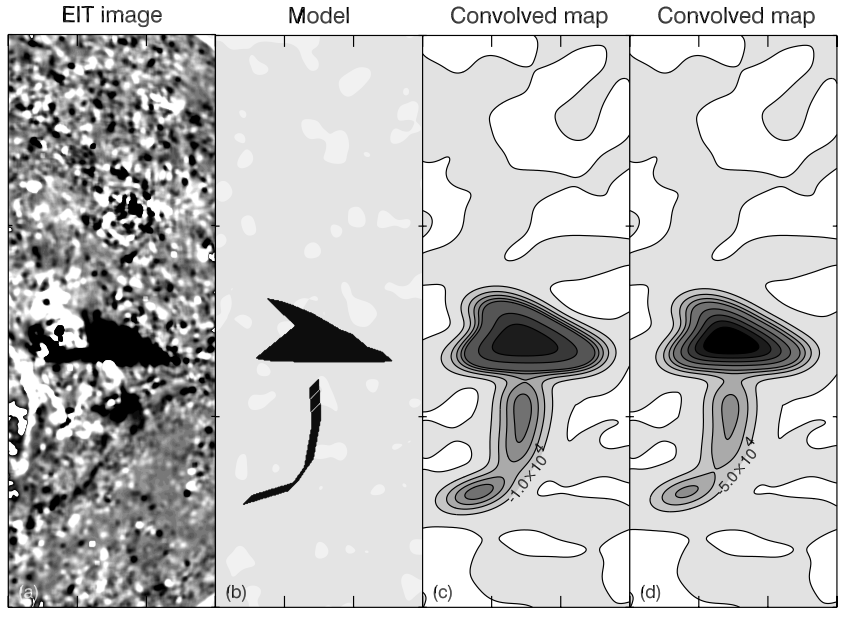

Fig. 8. Convolution of the EUV filament with a beam at $410.5 \mathrm{MHz}$. a) EIT difference image in the He II showing the erupting filament and the north depression (see Fig. 4). b) Model employed in the convolution, with a width of 10 pixels for the south part of the filament. Dark pattern were successively given to brightness temperatures of $7 \times 10^{5} \mathrm{~K}$ and $10^{5} \mathrm{~K}$. c) Convolved map with a filament brightness temperature of $7 \times 10^{5} \mathrm{~K}$; the mean temperature of the corona $\left(9 \times 10^{5} \mathrm{~K}\right)$ has been subtracted. The minimum of the depression within the southern feature is $\sim-5 \times 10^{4} \mathrm{~K}$. Contour levels are plotted at $[-2.5,-2,-1.5,-1,-0.5,-0.4,-0.3,-0.2,-0.1,0] \times$ $10^{5} \mathrm{~K} \mathrm{~d}$ ) ditto, with a filament brightness temperature of $10^{5} \mathrm{~K}$; the minimum of the depression within the southern feature is $\sim-1.6 \times 10^{5} \mathrm{~K}$. Contour levels are plotted at $[-7,-6,-5,-4,-3,-2,-1.5,-1,-0.5,0] \times 10^{5} \mathrm{~K}$.

be explained considering the global magnetic configuration. The north part lies in the vicinity of a strong magnetic flux coming from the nearby west active region. The delay between the departure time of dip F3 (Fig. 5) and the beginning of the event, which is about $40 \mathrm{~min}$, suggests that this magnetic flux holds up the expansion of the northern part of the filament. During the eruption, arcades are disturbed above the north site of the filament and the faint burst B that appears in their vicinity shows that F2 and F3 features interacted with the surrounding magnetic field. On the other hand, the south part can expand much more easily, as observed in radio wavelengths.

Finally, a possible scenario can be built up: a quiescent filament is embedded in a sheared magnetic structure (sigmoid) that is known to be potentially eruptive. A magnetic parasitic polarity appears near the north part of this structure, allowing reconnective processes in the vicinity of the filament seen as burst A. As the filament begins its slow ascension, the overlaying arcades are opening, and/or its surrounding magnetic structure inflates which leads to the radio depression. The south part expands freely into the outer corona, while the north one is hampered by the magnetic flux surrounding the small active region.

To conclude, this event illustrates the ability of radio investigations to study in detail the basic processes occurring during filament eruptions and CME onsets. Many signatures, currently observed in the EUV or SXR range, are now available in the long decimetric and metric radio 


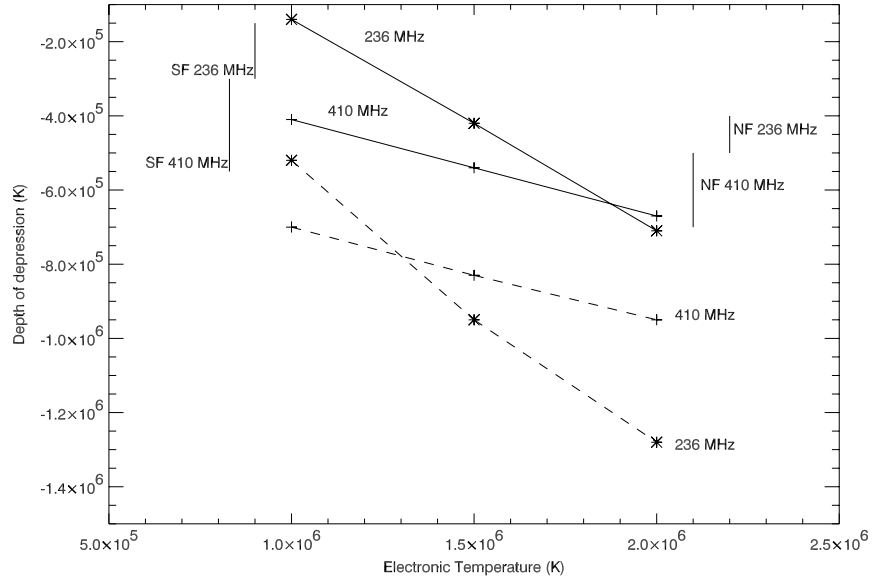

Fig. 9. Models of brightness temperature depression for a twofold (line) and a fourfold (dashed) drop in density, at two frequencies. The electronic temperature of the corona ranges from $10^{6}$ to $2 \times 10^{6} \mathrm{~K}$. Observations for the South part (SF) and the north part (NF) of the filament are given as a range of temperatures (vertical line), depending of the quiet sun level.

range at much higher altitudes. These kinds of events associated with faint energy releases are interesting because thermal radio observations are possible. The restructuring of the corona can be studied, indicated by dimming area around the site of the eruption, as well as the magnetic environment of the filament, probably its cavity. In addition, the faint non-thermal emissions give access to the time of particle accelerations, due to reconnection of magnetic field lines in the course of the event. In the future, these kinds of events may help to support theoretical analyses of eruptive phenomena in the corona.

Acknowledgements. The authors wish to thank K.-L. Klein and A. Kerdraon for useful discussions that lead to a significant improvement in the clarity of this paper. The LASCO data were provided by the Naval Research Laboratory (USA). LASCO was constructed by a consortium of the Naval Research Laboratory (USA), Max-Planck-Institut für Aeronomie (Germany), Laboratoire d'Astronomie Spatiale (France), and the University of Birmingham (UK). SOHO is a project of international cooperation between ESA and NASA. GOES data were provided by the Solar Data Analysis Center at NASA/GSFC. YOHKOH data used in this work were provided by the Yohkoh Data Archive Centre, at MSSL. The Nançay Radioheliograph is funded by the French Ministry of Education, the CNRS and the Région Centre.

\section{References}

Allen, C. W. 1947, MNRAS, 107, 426

Aulanier, G., \& Démoulin, P. 1998, A\&A, 329, 1125

Brueckner, G. E., Howard, R. A., Koomen, M. J., et al. 1995, Sol. Phys., 162, 357

Canfield, R. C., Hudson, H. S., \& Pevtsov, A. A. 2000, IEEE Trans. on Plasma Science, 28

Chen, P. F., \& Shibata, K. 2000, ApJ, 545, 524

Chiuderi Drago, F. 1990, in IAU Colloq. 117, Dynamics of Quiescent Prominences, 70

Delaboudinière, J. P., Artzner, G. E., Brunaud, J., et al. 1995, Sol. Phys., 162, 291

Delouis, J. 1999, Ph.D. Thesis, Université Paris VII

Engvold, O. 1988, in Solar and Stellar Coronal Structure and Dynamics, ed. E. Priest (Kluwer Academic Publishers), 151

Feynman, J., \& Martin, S. F. 1995, J. Geophys. Res., 100, 3355

Glover, A., Ranns, N. D. R., Harra, L. K., \& Culhane, J. L. 2000, Geophys. Res. Lett., 27, 2161

Harrison, R. A., \& Lyons, M. 2000, A\&A, 358, 1097

Howard, R. A., Sheeley, N. R., Michels, D. J., \& Koomen, M. J. 1985, J. Geophys. Res., 90, 8173

Hudson, H. S. 1999, Sol. Phys., 190, 91

Hudson, H. S., Acton, L. W., \& Freeland, S. L. 1996, ApJ, 470, 629

Hudson, H. S., Lemen, J. R. C. S., Sterling, A. C., \& Webb, D. F. 1998, Geophys. Res. Lett., 25, 2481

Kerdraon, A., \& Delouis, J. 1997, in Coronal Physics from Radio and Space Observations, ed. G. Trottet, 483

Marqué, C., Lantos, P., Klein, K., \& Delouis, J. M. 2001, A\&A, 374,316

Marqué, C., Lantos, P., Delouis, J., \& Alissandrakis, C. E. 1999, in Proc. 8th SOHO Workshop, ESA SP-446, 483

Pick, M. 1999, in Proceedings of the Nobeyama Symposium, held in Kiyosato, Japan, Oct. 27-30, 1998, ed. T. S. Bastian, N. Gopalswamy, \& K. Shibasaki, NRO Rep., 479, 187

Rust, D., \& Hildner, E. 1976, Sol. Phys., 48, 381

Rust, D. M., \& Kumar, A. 1996, ApJ, 464, L199

Saito, K., Poland, A. I., \& Munro, R. H. 1977, Sol. Phys., 55, 121

Saito, K., \& Tandberg-Hanssen, E. 1973, Sol. Phys., 31, 105

Sterling, A. C., Hudson, H. S., Thompson, B. J., \& Zarro, D. M. 2000, ApJ, 532, 628

Vaiana, G. S., Krieger, A. S., \& Timothy, A. F. 1973, Sol. Phys., 32, 81

Vourlidas, A., Subramanian, P., Dere, K., \& Howard, R. 2000, ApJ, 534, 456

Webb, D. F., \& Hundhausen, A. J. 1987, Sol. Phys., 108, 383 